\title{
WAVELET BASES FOR A UNITARY OPERATOR
}

\author{
by S. L. LEE, H. H. TAN and W. S. TANG
}

(Received 16th August 1993)

\begin{abstract}
Let $T$ be a unitary operator on a complex Hilbert space $\mathscr{H}$, and $X, Y$ be finite subsets of $\mathscr{H}$. We give a necessary and sufficient condition for $T^{\mathcal{Z}}(X):=\left\{T^{n} x: n \in \mathbf{Z}, x \in X\right\}$ to be a Riesz basis of its closed linear span $\left\langle T^{\mathrm{Z}}(X)\right\rangle$. If $T^{\mathrm{Z}}(X)$ and $T^{\mathrm{Z}}(Y)$ are Riesz bases, and $\left\langle T^{\mathrm{Z}}(X)\right\rangle \subset\left\langle T^{\mathrm{Z}}(Y)\right\rangle$, then $X$ is extendable to $X^{\prime}$ such that $T^{Z}\left(X^{\prime}\right)$ is a Riesz basis of $\left\langle T^{Z}(Y)\right\rangle$. The proof provides an algorithm for the construction of Riesz bases for the orthogonal complement of $\left\langle T^{\mathcal{Z}}(X)\right\rangle$ in $\left\langle T^{\mathrm{Z}}(Y)\right\rangle$. In the case $X$ consists of a single B-spline, the algorithm gives a natural and quick construction of the spline wavelets of Chui and Wang [2, 3]. Further, the duality principle of Chui and Wang in [3] and [4] is put in the general setting of biorthogonal Riesz bases in Hilbert space.
\end{abstract}

1991 Mathematics subject classification: 41A15, 41A30, 42C05, 42C15.

\section{Introduction}

Mallat [12] has introduced a general method for the construction of orthonormal wavelet bases via the multiresolution approximation, and Daubechies [5] has constructed an important class of compactly supported orthonormal wavelets, which are very efficient for numerical computations and image decomposition and reconstruction (see $[1,6])$.

Translates of a uniform B-spline form a Riesz basis of its closed linear span in $L^{2}(\mathbf{R})$, the space of square integrable functions. Orthonormalization of the B-spline basis leads to the Lemarié wavelets [11]. However the process does not preserve the simple and rich properties of uniform B-splines (see Schoenberg [15], and the references therein), which could be useful in practice. This has led Chui and Wang $([2,3,4])$ to consider cardinal spline wavelets which generate Riesz bases of their closed linear spans.

The spline wavelets of Chui and Wang are closely related to cardinal spline interpolation. In an attempt to construct spline wavelets based on cardinal Hermite interpolation, Goodman, Lee and Tang [8] have introduced wavelet bases generated by translating a finite set of functions. Coincidently, a result of Robertson [14] on wandering subspaces for unitary operators provides a general setting for orthonormal wavelets in Hilbert space. This provides a link between orthonormal wavelets and unitary operators, and the main object of this paper is to extend the link in order to provide a better perspective to spline wavelets, in particular the elegant results of Chui and Wang $[2,3]$.

In Section 2, we give a characterization of Riesz bases generated by a unitary operator $T$ on a finite set $Y$ in a Hilbert space. In Section 3, we show that if 
$T^{\mathbb{Z}}(Y):=\left\{T^{n} y: n \in Z, y \in Y\right\}$ is a Riesz basis of its closed linear span $\left\langle T^{\mathbb{Z}}(Y)\right\rangle$ and likewise, $T^{\mathbf{z}}(X)$ is a Riesz basis of $\left\langle T^{\mathbf{z}}(X)\right\rangle$, where $X$ is another finite set, and $\left\langle T^{\mathbf{z}}(X)\right\rangle \subset$ $\left\langle T^{\mathbf{Z}}(Y)\right\rangle$, then $X$ is extendable to $X^{\prime}$ such that $T^{\mathbf{Z}}\left(X^{\prime}\right)$ is a Riesz basis of $T^{\mathbf{Z}}(Y)$. Our proof is constructive, and provides an algorithm for the construction of Riesz bases for the orthogonal complement of $\left\langle T^{\mathrm{Z}}(X)\right\rangle$ in $\left\langle T^{\mathrm{Z}}(Y)\right\rangle$. This result is analogous to the main theorem of Robertson [14]. Some identities related to duality are also obtained in Section 3. Section 4 deals with Riesz bases of wavelets in Hilbert space. We extend the duality principle of [3] and [4] to the general setting of biorthogonal Riesz bases in Hilbert space. Explicit construction of Riesz bases for the orthogonal complement of $T^{\mathrm{Z}}(X)$ in $T^{\mathrm{Z}}(Y)$ is given in the case $X$ consists of one vector. In Section 5 we show how the spline wavelets of Chui and Wang are derived naturally from the general theory. Spline wavelets can be useful in numerical computation because of the availability of the cardinal interpolant and the ease with which B-spline series can be evaluated. The problem of matrix compression by wavelets is discussed in the last section.

Throughout this paper, the inner product of any two vectors $x$ and $y$ in a Hilbert space is denoted by $\langle x, y\rangle$. We let $\tilde{L}^{2}(0,2 \pi)$ be the space of all square integrable $2 \pi$-periodic complex-valued functions on the real line $\mathbf{R}$, and let $\tilde{L}_{s}^{2}(0,2 \pi)$ (respectively $\tilde{L}_{m \times s}^{2}(0,2 \pi)$ ) be the set of all row vectors with $s$ components (respectively all $m \times s$ matrices with entries) in $\tilde{L}^{2}(0,2 \pi)$. We denote by $I_{m}$ the $m \times m$ identity matrix. Given two Hermitian $m \times m$ matrices $A$ and $B$, we write $A \leqq B$ if the matrix $B-A$ is positive semidefinite.

\section{Characterization of Riesz bases for a unitary operator}

Let $T$ be a unitary operator on a complex Hilbert space $\mathscr{H}$. For $V \subset \mathscr{H}$ we shall write

$$
T^{\mathrm{Z}}(V):=\left\{T^{n} v: n \in \mathbf{Z}, v \in V\right\},
$$

and let $\left\langle T^{\mathrm{z}}(V)\right\rangle$ denote the closed linear span of $T^{\mathrm{z}}(V)$.

Let $Y=\left\{y_{1}, \ldots, y_{s}\right\} \subset \mathscr{H}$, and suppose that

$$
\left(\left\langle y_{k}, T^{n} y_{j}\right\rangle\right)_{n \in \mathbf{Z}} \in l^{2}(Z), \quad k, j=1, \ldots, s .
$$

Then

$$
\sum_{n \in \mathbf{Z}}\left\langle y_{k}, T^{n} y_{l}\right\rangle e^{i n \theta} \in \tilde{L}^{2}(0,2 \pi)
$$

for $k, l=1, \ldots, s$. Let

$$
\Phi_{Y}(\theta):=\left(\sum_{n \in \mathbf{Z}}\left\langle y_{k}, T^{n} y_{l}\right\rangle e^{i n \theta}\right)_{k, l=1}^{s}
$$

Then for almost all $\theta, \Phi_{Y}(\theta)$ is a Hermitian matrix. Let $\lambda_{j}(\theta), j=1, \ldots, s$, be its 
eigenvalues. The main result in this section is the characterization of $T^{\mathbf{Z}}(\eta)$ as a Riesz basis of its closed linear span.

Theorem 2.1. The set $T^{\mathrm{Z}}(Y)$ is a Riesz basis of $\left\langle T^{\mathrm{Z}}(Y)\right\rangle$ if and only if there are positive constants $C_{1}$ and $C_{2}$ such that

$$
C_{1} \leqq \lambda_{j}(\theta) \leqq C_{2}
$$

for almost all $\theta$ and for $j=1, \ldots, s$, or equivalently, $C_{1} I_{s} \leqq \Phi_{Y}(\theta) \leqq C_{2} I_{s}$ for almost all $\theta$.

To set up the proof of Theorem 2.1 we shall first consider some related results.

Lemma 2.1. Suppose $T^{\mathrm{Z}}(Y)$ is a Riesz basis of $\left\langle T^{\mathrm{Z}}(Y)\right\rangle$ and let $F:\left\langle T^{\mathrm{Z}}(Y)\right\rangle \rightarrow\left\langle T^{\mathrm{Z}}(Y)\right\rangle$ be defined by

$$
F(v):=\sum_{j=1}^{s} \sum_{n \in Z}\left\langle v, T^{n} y_{j}\right\rangle T^{n} y_{j}, \quad v \in\left\langle T^{\mathrm{Z}}(Y)\right\rangle
$$

Then $F$ is a positive, bounded invertible operator which commutes with $T$.

Proof. The fact that $F$ is positive, bounded and invertible is well-known (see [16, p. 185]). The commutativity of $F$ and $T$ follows by applying (2.3) to $F T(v)$, by virtue of the fact that $T$ is unitary.

For any finite set $Y=\left\{y_{1}, \ldots, y_{s}\right\} \subset \mathscr{H}$ such that $T^{\mathrm{Z}}(Y)$ is a Riesz basis of $\left\langle T^{\mathrm{Z}}(Y)\right\rangle$, we define

$$
\tilde{Y}:=F^{-1}(Y),
$$

and denote

$$
\tilde{y}_{j}=F^{-1}\left(y_{j}\right), \quad j=1, \ldots, s .
$$

By Lemma 2.1

$$
T^{n} \tilde{y}_{j}=\Gamma^{-1} T^{n} y_{j}, \quad n \in \mathbf{Z}, j=1, \ldots, s .
$$

Equations (2.3) and (2.4) give

$$
y_{k}=\sum_{j=1}^{s} \sum_{n \in \mathbf{Z}}\left\langle\tilde{y}_{k}, T^{n} y_{j}\right\rangle T^{n} y_{j}
$$

from which we obtain 


$$
\left\langle\tilde{y}_{k}, T^{n} y_{j}\right\rangle=\delta_{0, n} \delta_{k, j}, \quad n \in \mathbf{Z}, k, j=1, \ldots, s .
$$

Thus $T^{\mathbf{Z}}(Y)$ and $T^{\mathbf{Z}}(\tilde{Y})$ are biorthogonal Riesz bases of $\left\langle T^{\mathbf{Z}}(Y)\right\rangle$. Any $v \in\left\langle T^{\mathbf{Z}}(Y)\right\rangle$ can be written as

$$
\begin{aligned}
v & =\sum_{j=1}^{s} \sum_{n \in \mathbf{Z}}\left\langle v, T^{n} \tilde{y}_{j}\right\rangle T^{n} y_{j} \\
& =\sum_{j=1}^{s} \sum_{n \in \mathbf{Z}}\left\langle v, T^{n} y_{j}\right\rangle T^{n} \tilde{y}_{j} .
\end{aligned}
$$

Proposition 2.1. For any finite set $Y \subset \mathscr{H}, \boldsymbol{\Phi}_{\mathbf{Y}}(\theta)$ is positive semi-definite for almost all $\theta$.

Proof. Let $\theta \in \mathbf{R}$ and $A=\left(\alpha_{j}\right)_{j=1}^{s} \in \mathbf{C}^{s}$. Then

$$
A \Phi_{Y}(\theta) A^{*}=\sum_{n \in \mathbf{Z}}\left\langle v, T^{n} v\right\rangle e^{i n \theta}
$$

where

$$
v:=\sum_{j=1}^{s} \alpha_{j} y_{j}
$$

Since $\left(\left\langle y_{k}, T^{n} y_{l}\right\rangle\right)_{n \in Z} \in l^{2}(Z)$ for $k, l=1, \ldots, s$, so is the sequence $\left(\left\langle v, T^{n} v\right\rangle\right)_{n \in Z}$. Hence the expression on the right of (2.6) defines a function $f$ in $\tilde{L}^{2}(0,2 \pi)$ with Fourier coefficients $\hat{f}(n)=\left\langle v, T^{n} v\right\rangle, n \in \mathbf{Z}$. We shall show that $f$ is nonnegative. Indeed for any finite sequence $\left(c_{n}\right)$ of complex numbers,

$$
\begin{aligned}
\sum_{m, n} \hat{f}(n-m) c_{m} \bar{c}_{n} & =\sum_{m, n}\left\langle T^{m} v, T^{n} v\right\rangle c_{m} \bar{c}_{n} \\
& =\left\|\sum_{n} c_{n} T^{n} v\right\|^{2} \geqq 0
\end{aligned}
$$

Therefore $(\hat{f}(n))_{n \in Z}$ is a positive definite sequence. By a well-known theorem in Fourier analysis $([7$, p. 116$]), f$ is nonnegative almost everywhere.

We need a property that is weaker than that of a Riesz basis.

Lemma 2.2. Let $\left(f_{n}\right)_{n \in \mathrm{Z}}$ be a sequence in $\mathscr{H}$. The following conditions are equivalent. 
(1)

$$
\sum_{n \in \mathbf{Z}}\left|\left\langle f, f_{n}\right\rangle\right|^{2}<\infty \quad \text { for every } f \in \mathscr{H}
$$

(2) There exists a positive constant $M$ such that

$$
\sum_{n \in Z}\left|\left\langle f, f_{n}\right\rangle\right|^{2} \leqq M\|f\|^{2} \quad \text { for every } f \in \mathscr{H}
$$

(3) There exists a positive constant $M$ such that

$$
\left\|\sum c_{n} f_{n}\right\|^{2} \leqq M \sum\left|c_{n}\right|^{2}
$$

for every finite sequence $\left(c_{n}\right)$ of scalars.

(4) There exists a positive constant $M$ such that

$$
\left\|\sum_{n \in Z} c_{n} f_{n}\right\|^{2} \leqq M \sum_{n \in Z}\left|c_{n}\right|^{2}
$$

for all $\left(c_{n}\right)_{n \in Z} \in l^{2}(Z)$.

(5) $\sum_{n \in \mathbf{Z}} c_{n} f_{n}$ converge in $\mathscr{H}$ for every $\left(c_{n}\right)_{n \in Z} \in l^{2}(Z)$.

Proof. The proof of the equivalence of conditions (1), (2) and (3) can be found in [16, p. 154-155]. Suppose that (3) holds. If $\left(c_{n}\right)_{n \in Z} \in l^{2}(Z)$, then (3) implies that the partial sums of $\sum_{n \in Z} c_{n} f_{n}$ form a Cauchy sequence in $\mathscr{H}$. Therefore (5) holds. Taking limits in the inequality in (3) gives (4). The implication $(4) \Rightarrow(3)$ is obvious. It remains to prove that $(5) \Rightarrow(1)$.

Fix $f \in \mathscr{H}$. Take any $\left(c_{n}\right)_{n \in Z} \in l^{2}(\mathbf{Z})$. For any $n \in \mathbf{Z}$, choose $\theta_{n} \in[0,2 \pi)$ so that

$$
c_{n}\left\langle f, f_{n}\right\rangle=\left|c_{n}\left\langle f, f_{n}\right\rangle\right| e^{i \theta_{n}}
$$

By (5), the series $\sum_{n \in \mathrm{Z}} \bar{c}_{n} e^{i \theta_{n}} f_{n}$ converges to some vector $g$ in $\mathscr{H}$, and

$$
\sum_{n \in \mathbf{Z}}\left|c_{n}\left\langle f, f_{n}\right\rangle\right|=\sum_{n \in \mathbf{Z}} c_{n} e^{-i \theta_{n}}\left\langle f, f_{n}\right\rangle=\langle f, g\rangle<\infty
$$

Hence $\left(\left\langle f, f_{n}\right\rangle\right)_{n \in Z} \in l^{2}(Z)$.

Remark 1. A sequence $\left(f_{n}\right)_{n \in Z}$ in $\mathscr{H}$ satisfying condition (1) in Lemma 2.2 is called a Bessel sequence (see $\left[16\right.$, p. 154]). Under the assumption that $\left(f_{n}\right)_{n \in Z}$ is a Schauder basis of $\mathscr{H}$, the equivalence of conditions (3) and (5) can be found in [16, p. 37].

We next give a sufficient condition for a sequence $\left(T^{n} x\right)_{n \in Z}, x \in \mathscr{H}$, to satisfy the conditions of Lemma 2.2 . 
Lemma 2.3. Let $x \in \mathscr{H}$. Suppose that $\left(\left\langle x, T^{n} x\right\rangle\right)_{n \in Z}$ is in $l^{2}(Z)$ and there exists a positive constant $B$ such that

$$
\sum_{n \in \mathbf{Z}}\left\langle x, T^{n} x\right\rangle e^{i n \theta} \leqq B
$$

for almost all $\theta$. Then

$$
\left\|\sum c_{n} T^{n} x\right\|^{2} \leqq B \sum\left|c_{n}\right|^{2}
$$

for every finite sequence $\left(c_{n}\right)$ of scalars.

Proof. Let $\Phi(\theta)=\sum_{n \in Z}\left\langle x, T^{n} x\right\rangle e^{i n \theta}$. By Proposition 2.1, $\Phi(\theta) \geqq 0$ for almost all $\theta$. Let $v=\sum c_{l} T^{l} x$, where $\left(c_{l}\right)$ is a finite sequence. Then

$$
\left\langle v, T^{n} x\right\rangle=\sum c_{l}\left\langle x, T^{n-l} x\right\rangle, \quad n \in \mathbf{Z}
$$

Hence $\left(\left\langle v, T^{n} x\right\rangle\right)_{n \in Z} \in l^{2}(Z)$,

$$
\sum_{n \in Z}\left\langle v, T^{n} x\right\rangle e^{i n \theta}=\left(\sum c_{n} e^{i n \theta}\right) \Phi(\theta) \quad \text { a.e. }
$$

and

$$
\sum_{n \in \mathbf{Z}}\left|\left\langle v, T^{n} x\right\rangle\right|^{2}=\frac{1}{2 \pi} \int_{-\pi}^{\pi}\left|\sum c_{n} e^{i n \theta}\right|^{2}|\Phi(\theta)|^{2} d \theta \leqq B^{2} \sum\left|c_{n}\right|^{2}
$$

Then

$$
\begin{aligned}
\left\|v^{2}\right\| & =\langle v, v\rangle=\sum \bar{c}_{n}\left\langle v, T^{n} x\right\rangle \\
& \leqq\left(\sum\left|c_{n}\right|^{2}\right)^{1 / 2}\left(\sum\left|\left\langle v, T^{n} x\right\rangle\right|^{2}\right)^{1 / 2} \\
& \leqq B \sum\left|c_{n}\right|^{2} .
\end{aligned}
$$

Proof of Theorem 2.1. Let $\left(a_{n}^{j}\right)_{n \in Z} \in l^{2}(Z), j=1, \ldots, s$, and suppose that

$$
v:=\sum_{j=1}^{s} \sum_{n \in Z} a_{n}^{j} T^{n} y_{j}
$$

converges in $\mathscr{H}$ and $\left(\left\langle v, T^{n} y_{j}\right\rangle\right)_{n \in Z} \in l^{2}(Z), j=1, \ldots, s$. Let

$$
\begin{gathered}
A_{j}(\theta):=\sum_{n \in \mathbf{Z}} a_{n}^{j} e^{i n \theta}, \\
V_{j}(\theta):=\sum_{n \in \mathbf{Z}}\left\langle v, T^{n} y_{j}\right\rangle e^{i n \theta}, \quad j=1, \ldots, s
\end{gathered}
$$


$A:=\left(A_{j}\right)_{j=1}^{s}$ and $V:=\left(V_{j}\right)_{j=1}^{s} \in \tilde{L}_{s}^{2}(0,2 \pi)$. Then

$$
V=A \Phi_{Y}
$$

Let $U$ be the unitary matrix such that

$$
U^{*} \boldsymbol{\Phi}_{Y} U=D=\operatorname{diag}\left(\lambda_{1}, \ldots, \lambda_{s}\right)
$$

By (2.7),

$$
V V^{*}=B D^{2} B^{*}
$$

where

$$
B=\left(B_{j}\right)_{j=1}^{s}:=A U
$$

Integrating (2.8) gives

$$
\sum_{j=1}^{s}\left\|V_{j}\right\|^{2}=\frac{1}{2 \pi} \int_{0}^{2 \pi} \sum_{j=1}^{s} \lambda_{j}(\theta)^{2}\left|B_{j}(\theta)\right|^{2} d \theta
$$

Suppose $T^{\mathbf{Z}}(Y)$ is a Riesz basis of $\left\langle T^{\mathbf{Z}}(Y)\right\rangle$ and let $T^{\mathbf{Z}}(\tilde{Y})$ be the dual basis. Then there are positive constants $K, L, \tilde{K}, \tilde{L}$ such that for any sequences $\left(a_{n}^{j}\right)_{n \in \mathbf{Z}} \in l^{2}(Z)$, $j=1, \ldots, s$, the vector

$$
\begin{aligned}
v: & =\sum_{j=1}^{s} \sum_{n \in \mathbf{Z}} a_{n}^{j} T^{n} y_{j} \\
& =\sum_{j=1}^{s} \sum_{n \in \mathbf{Z}}\left\langle v, T^{n} y_{j}\right\rangle T^{n} \tilde{y}_{j},
\end{aligned}
$$

satisfies

$$
K \sum_{j=1}^{s} \sum_{n \in Z}\left|a_{n}^{j}\right|^{2} \leqq\|v\|^{2} \leqq L \sum_{j=1}^{s} \sum_{n \in Z}\left|a_{n}^{j}\right|^{2}
$$

and

$$
\tilde{K} \sum_{j=1}^{s} \sum_{n \in \mathbf{Z}}\left|\left\langle v, T^{n} y_{j}\right\rangle\right|^{2} \leqq\|v\|^{2} \leqq\left.\tilde{L} \sum_{j=1}^{s} \sum_{n \in \mathbf{Z}}\left|v, T^{n} y_{j}\right\rangle\right|^{2}
$$

Since $\sum_{j=1}^{s}\left\|V_{j}\right\|^{2}=\sum_{j=1}^{s} \sum_{n \in Z}\left|\left\langle v, T^{n} y_{j}\right\rangle\right|^{2}$, it follows from (2.9), (2.10) and (2.11) that

$$
\frac{K}{\widetilde{L}} \sum_{j=1}^{s} \sum_{n \in Z}\left|a_{n}^{j}\right|^{2} \leqq \frac{1}{2 \pi} \int_{0}^{2 \pi} \sum_{j=1}^{s} \lambda_{j}(\theta)^{2}\left|B_{j}(\theta)\right|^{2} d \theta \leqq \frac{L}{\widetilde{K}} \sum_{j=1}^{s} \sum_{n \in Z}\left|a_{n}^{j}\right|^{2} .
$$


But

$$
\sum_{j=1}^{s} \sum_{n \in Z}\left|a_{n}^{j}\right|^{2}=\sum_{j=1}^{s}\left\|A_{j}\right\|^{2}=\sum_{j=1}^{s}\left\|B_{j}\right\|^{2}
$$

which, by (2.12), gives

$$
\left.\frac{K}{\widetilde{L}} \sum_{j=1}^{s} \int_{0}^{2 \pi}\left|B_{j}(\theta)\right|^{2} d \theta \leqq \sum_{j=1}^{s} \int_{0}^{2 \pi} \lambda_{j}(\theta)^{2} \mid B_{j}(\theta)\right)\left.\right|^{2} d \theta \leqq \frac{L}{\widetilde{K}} \sum_{j=1}^{s} \int_{0}^{2 \pi}\left|B_{j}(\theta)\right|^{2} d \theta .
$$

The inequalities hold for all $B_{j} \in \tilde{L}^{2}(0,2 \pi), j=1, \ldots, s$. Hence

$$
\frac{K}{\widetilde{L}} \leqq \lambda_{j}(\theta)^{2} \leqq \frac{L}{\widetilde{K}}
$$

for almost all $\theta$. Since $\lambda_{j}(\theta) \geqq 0$, we obtain (2.2).

Conversely, suppose that (2.2) holds. Let $\Phi_{j}(\theta):=\sum_{n \in Z}\left\langle y_{j}, T^{n} y_{j}\right\rangle e^{i n \theta}$ be the $(j, j)$-entry of the matrix $\Phi_{Y}(\theta), j=1, \ldots, s$. Let $U(\theta)$ be the unitary matrix such that $\Phi_{Y}(\theta)=$ $U(\theta) \operatorname{diag}\left(\lambda_{1}(\theta), \ldots, \lambda_{s}(\theta)\right) U(\theta)^{*}$. Then for $j=1, \ldots, s$,

$$
\boldsymbol{\Phi}_{j}(\theta)=e_{j} \Phi_{Y}(\theta) e_{j}^{*}=\sum_{k=1}^{s} \lambda_{k}(\theta)\left|U_{j k}(\theta)\right|^{2}
$$

where $e_{j}$ is the row vector $\left(\delta_{j k}\right)_{k=1}^{s}$ and $\left(U_{j k}(\theta)\right)_{k=1}^{s}$ is the $j$ th row of $U(\theta)$. Since $\sum_{k=1}^{s}\left|U_{j k}(\theta)\right|^{2}=1$, by (2.2)

$$
\boldsymbol{\Phi}_{j}(\theta) \leqq C_{2} \quad \text { a.e. for } j=1, \ldots, s \text {. }
$$

By Lemma 2.3 and Lemma 2.2,

$$
\sum_{n \in \mathbf{Z}}\left|\left\langle v, T^{n} y_{j}\right\rangle\right|^{2}<\infty, \quad v \in \mathscr{H}, j=1, \ldots, s
$$

and $\sum_{n \in \mathbf{Z}} a_{n}^{j} T^{n} y_{j}$ converges in $\mathscr{H}$ for $\left(a_{n}^{j}\right)_{n \in \mathbf{Z}} \in l^{2}(Z), j=1, \ldots, s$.

By (2.9), we have for any

$$
\begin{gathered}
v=\sum_{j=1}^{s} \sum_{n \in \mathbf{Z}} a_{n}^{j} T^{n} y_{j}, \quad\left(a_{n}^{j}\right)_{n \in \mathbf{Z}} \in l^{2}(\mathbf{Z}), j=1, \ldots, s, \\
C_{1}^{2} \sum_{j=1}^{s} \sum_{n \in \mathbf{Z}}\left|a_{n}^{j}\right|^{2} \leqq \sum_{j=1}^{s}\left\|V_{j}\right\|^{2} \leqq C_{2}^{2} \sum_{j=1}^{s} \sum_{n \in \mathbf{Z}}\left|a_{n}^{j}\right|^{2}
\end{gathered}
$$

Now, 


$$
\begin{aligned}
\|v\|^{2} & =\langle v, v\rangle \\
& =\sum_{j=1}^{s} \sum_{n \in Z} \bar{a}_{n}^{j}\left\langle v, T^{n} y_{j}\right\rangle \\
& \leqq\left(\sum_{j=1}^{s} \sum_{n \in Z}\left|a_{n}^{j}\right|^{2}\right)^{1 / 2}\left(\sum_{j=1}^{s} \sum_{n \in Z}\left|\left\langle v, T^{n} y_{j}\right\rangle\right|^{2}\right)^{1 / 2} \\
& =\left(\sum_{j=1}^{s} \sum_{n \in Z}\left|a_{n}^{j}\right|^{2}\right)^{1 / 2}\left(\sum_{j=1}^{s}\left\|V_{j}\right\|^{2}\right)^{1 / 2},
\end{aligned}
$$

and applying (2.14) leads to

$$
\|v\|^{2} \leqq C_{2} \sum_{j=1}^{s} \sum_{n \in \mathbf{Z}}\left|a_{n}^{j}\right|^{2}
$$

On the other hand,

$$
\begin{gathered}
\sum_{j=1}^{s} \sum_{n \in \mathbf{Z}}\left|\left\langle v, T^{n} y_{j}\right\rangle\right|^{2}=\left\langle v, \sum_{j=1}^{s} \sum_{n \in \mathbf{Z}}\left\langle v, T^{n} y_{j}\right\rangle T^{n} y_{j}\right\rangle \\
\leqq\|v\|\left\|\sum_{j=1}^{s} \sum_{n \in \mathbf{Z}}\left\langle v, T^{n} y_{j}\right\rangle T^{n} y_{j}\right\| .
\end{gathered}
$$

By (2.15)

$$
\left\|\sum_{j=1}^{s} \sum_{n \in Z}\left\langle v, T^{n} y_{j}\right\rangle T y_{j}\right\| \leqq \sqrt{C_{2}}\left(\sum_{j=1}^{s} \sum_{n \in Z}\left|\left\langle v, T^{n} y_{j}\right\rangle\right|^{2}\right)^{1 / 2}
$$

which together with (2.16) leads to

$$
\begin{aligned}
\|v\|^{2} & \geqq \frac{1}{C_{2}}\left(\sum_{j=1}^{s} \sum_{n \in \mathbf{Z}}\left|\left\langle v, T^{n} y_{j}\right\rangle\right|^{2}\right) \\
& =\frac{1}{C_{2}} \sum_{j=1}^{s}\left\|V_{j}\right\|^{2}
\end{aligned}
$$

Combining the first inequality in (2.14) and (2.17) gives 


$$
\|v\|^{2} \geqq \frac{C_{1}^{2}}{C_{2}} \sum_{j=1}^{s} \sum_{n \in Z}\left|a_{n}^{j}\right|^{2} .
$$

The inequalities (2.15) and (2.18) show that $\left\{T^{n} y_{j}: n \in Z, j=1, \ldots, s\right\}$ is a Riesz basis of $\left\langle T^{\mathrm{Z}}(Y)\right\rangle$.

Proposition 2.2. Suppose $T^{\mathrm{Z}}(Y)$ and $T^{\mathrm{Z}}(\tilde{Y})$ are biorthogonal Riesz bases of $\left\langle T^{\mathrm{Z}}(Y)\right\rangle$. Then

$$
\Phi_{Y}^{-1}=\Phi_{\mathbf{Y}}
$$

Proof. For $v \in \mathbf{Z}$ and $k, l=1, \ldots, s$,

$$
\begin{aligned}
\delta_{0 . v} \delta_{k, l} & =\left\langle\tilde{y}_{k}, T^{\vee} y_{l}\right\rangle \\
& =\left\langle\tilde{y}_{k}, \sum_{j=1}^{s} \sum_{n \in Z}\left\langle T^{v} y_{l}, T^{n} y_{j}\right\rangle T^{n} \tilde{y}_{j}\right\rangle \\
& =\sum_{j=1}^{s} \sum_{n \in Z}\left\langle\tilde{y}_{k}, T^{n} \tilde{y}_{j}\right\rangle \overline{\left\langle T^{v-n} y_{l}, y_{j}\right\rangle}
\end{aligned}
$$

Now,

$$
\begin{array}{r}
\sum_{j=1}^{s}\left(\sum_{v \in \mathbf{Z}}\left\langle\tilde{y}_{k}, T^{v} \tilde{y}_{j}\right\rangle e^{i v \theta}\right)\left(\overline{\sum_{v \in \mathbf{Z}}\left\langle y_{l}, T^{v} y_{j}\right\rangle e^{i v \theta}}\right) \\
=\sum_{j=1}^{s}\left(\sum_{v \in \mathbf{Z}}\left\langle\tilde{y}_{k}, T^{v} \tilde{y}_{j}\right\rangle e^{i v \theta}\right)\left(\sum_{v \in \mathbf{Z}} \overline{\left\langle T^{v} y_{l}, y_{j}\right\rangle} e^{i v \theta}\right) \\
=\sum_{j=1}^{s} \sum_{v \in \mathbf{Z}} \sum_{n \in \mathbf{Z}}\left\langle\tilde{y}_{k}, T^{n} \tilde{y}_{j}\right\rangle \overline{\left\langle T^{v-n} y_{l}, y_{j}\right\rangle} e^{i v \theta}=\delta_{k, l},
\end{array}
$$

which shows that

$$
\boldsymbol{\Phi}_{P} \boldsymbol{\Phi}_{Y}^{*}=I
$$

The result follows since $\boldsymbol{\Phi}_{\boldsymbol{y}}$ is Hermitian.

\section{Extension of Riesz bases for a unitary operator}

Throughout this section, let $X=\left\{x_{1}, \ldots, x_{r}\right\}$ and $Y=\left\{y_{1}, \ldots, y_{s}\right\}$ be finite subsets of $\mathscr{H}$ and suppose $T^{\mathrm{z}}(X)$ and $T^{\mathrm{z}}(Y)$ are Riesz bases of $\left\langle T^{\mathrm{z}}(X)\right\rangle$ and $\left\langle T^{\mathrm{Z}}(Y)\right\rangle$ respectively. 
Our main results here are the following analogues of Robertson's theorems [14] on wandering subspaces for unitary operators.

Theorem 3.1. Suppose $s=r$ and $\left\langle T^{\mathrm{z}}(X)\right\rangle \subset\left\langle T^{\mathrm{z}}(Y)\right\rangle$. Then $\left\langle T^{\mathrm{z}}(X)\right\rangle=\left\langle T^{\mathrm{Z}}(Y)\right\rangle$.

Theorem 3.2. If $\left\langle T^{\mathbb{Z}}(X)\right\rangle$ is a proper subset of $\left\langle T^{\mathbb{Z}}(Y)\right\rangle$, then there exists $x_{r+1} \in\left\langle T^{\mathbb{Z}}(Y)\right\rangle, \quad x_{r+1} \perp\left\langle T^{\mathbb{Z}}(X)\right\rangle$ such that $T^{\mathbf{Z}}\left(X \cup\left\{x_{r+1}\right\}\right)$ is a Riesz basis of $\left\langle T^{\mathbf{z}}\left(X \cup\left\{x_{r+1}\right\}\right)\right\rangle$.

Theorems 3.1 and 3.2 can be proved by applying Robertson's theorems and a modified form of the Fuglede-Putnam theorem ([8, Lemma 2.1]). However, we shall prove them directly among other related results as they are of independent interest.

Since $T^{\mathrm{Z}}(X)$ and $T^{\mathrm{Z}}(Y)$ are Riesz bases of their respective closed linear spans, there exist $\tilde{X}=\left\{\tilde{x}_{1}, \ldots \tilde{x}_{r}\right\} \subset\left\langle T^{\mathbb{Z}}(X)\right\rangle$ and $\tilde{Y}=\left\{\tilde{y}_{1}, \ldots, \tilde{y}_{s}\right\} \subset\left\langle T^{\mathbb{Z}}(Y)\right\rangle$ such that

$$
\begin{array}{cl}
\left\langle\tilde{x}_{k}, T^{v} x_{l}\right\rangle=\delta_{0, v} \delta_{k, l}, & v \in \mathbf{Z}, k, l=1, \ldots, r, \\
\left\langle\tilde{y}_{m}, T^{v} y_{n}\right\rangle=\delta_{0, v} \delta_{m, n}, & v \in \mathbf{Z}, m, n=1, \ldots, s .
\end{array}
$$

Therefore, $T^{\mathrm{Z}}(\tilde{X})$ and $T^{\mathrm{z}}(X)$ are biorthogonal Riesz bases of $\left\langle T^{\mathrm{Z}}(X)\right\rangle$, and $T^{\mathrm{Z}}(\tilde{Y})$ and $T^{\mathbf{Z}}(Y)$ are biorthogonal Riesz bases of $\left\langle T^{\mathbf{Z}}(Y)\right\rangle$.

For any subset $V=\left\{v_{1}, \ldots, v_{m}\right\}$ of $\left\langle T^{\mathbb{Z}}(Y)\right\rangle$, where $1 \leqq m \leqq s$, let

$$
\begin{aligned}
& p_{k, l}^{V}(\theta):=\sum_{n \in Z}\left\langle v_{k}, T^{n} y_{l}\right\rangle e^{i n \theta} \\
& \tilde{p}_{k, l}^{V}(\theta):=\sum_{n \in \mathbf{Z}}\left\langle v_{k}, T^{n} \tilde{y}_{l}\right\rangle e^{i n \theta}
\end{aligned}
$$

for $k=1, \ldots, m, l=1, \ldots, s$, and

$$
P_{V}=\left(p_{k, 1}^{V}\right)_{m \times s^{\prime}}, \quad \tilde{P}_{V}=\left(\tilde{p}_{k, 1}^{V}\right)_{m \times s} .
$$

Then $P_{V}, \tilde{P}_{V} \in \tilde{L}_{m \times s}^{2}(0,2 \pi)$.

Proposition 3.1. For any $V=\left\{v_{1}, \ldots, v_{m}\right\} \subset\left\langle T^{\mathbf{Z}_{(}}(Y)\right\rangle$,

$$
P_{V}=\tilde{P}_{\boldsymbol{V}} \boldsymbol{\Phi}_{\boldsymbol{Y}}
$$

If in addition $\left(\left\langle v_{k}, T^{v} v_{l}\right\rangle\right)_{v \in \mathbf{Z}} \in l^{2}(\mathbf{Z}), k, l=1, \ldots, m$, then

$$
\tilde{\boldsymbol{P}}_{V} P_{V}^{*}=\boldsymbol{\Phi}_{V} .
$$

Proof. The equation (3.3) is proved in the same manner as Proposition 2.2, using 


$$
\left\langle v_{k}, T^{v} y_{l}\right\rangle=\sum_{j=1}^{s} \sum_{n \in \mathbb{Z}}\left\langle v_{k}, T^{n} \tilde{y}_{j}\right\rangle \overline{\left\langle T^{v} y_{l}, T^{n} y_{j}\right\rangle},
$$

whereas (3.4) is obtained using

$$
\begin{aligned}
\left\langle v_{k}, T^{v} v_{l}\right\rangle & =\left\langle v_{k}, \sum_{j=1}^{s} \sum_{n \in \mathbf{Z}}\left\langle T^{v} v_{l}, T^{n} y_{j}\right\rangle T^{n} \tilde{y}_{j}\right\rangle \\
& =\sum_{j=1}^{s} \sum_{n \in \mathbf{Z}}\left\langle v_{k}, T^{n} \tilde{y}_{j}\right\rangle \overline{\left\langle T^{v} v_{l}, T^{n} y_{j}\right\rangle}
\end{aligned}
$$

Proposition 3.2. If $\left\langle T^{\mathrm{z}}(X)\right\rangle \subset\left\langle T^{\mathrm{z}}(Y)\right\rangle$, then

$$
\tilde{P}_{\hat{X}} P_{X}^{*}=P_{X} \tilde{P}_{X}^{*}=I_{r}
$$

where $I_{r}$ is the $r \times r$ identity matrix.

Proof. The proof is the same as before, using (3.1). We shall omit the details. and

Corollary 3.1. If $\left\langle T^{\mathrm{z}}(X)\right\rangle \subset\left\langle T^{\mathrm{z}}(Y)\right\rangle$ and $r=s$, then $P_{X}, P_{X}, \tilde{P}_{X}$ and $\tilde{P}_{X}$ are invertible,

$$
P_{X}^{-1}=\tilde{P} \frac{k}{x}, \quad P_{\bar{X}}^{-1}=\tilde{P}_{x}^{*}
$$

Furthermore,

$$
\begin{aligned}
& P_{P}^{*} P_{X}=\Phi_{Y}, \\
& \tilde{P}_{X}^{*} \tilde{P}_{X}=\Phi_{Y} .
\end{aligned}
$$

Proof. If $r=s$, (3.6) follows from (3.5). By (3.3) and 3.6

$$
\begin{aligned}
\boldsymbol{P}_{X} & =\tilde{P}_{X} \Phi_{Y} \\
& =\left(P_{X}^{*}\right)^{-1} \Phi_{Y},
\end{aligned}
$$

which gives (3.7). Finally, (3.8) follows from (3.7), (2.19) and (3.6).

Corollary 3.2. If $\left\langle T^{\mathrm{z}}(X)\right\rangle \subset\left\langle T^{\mathrm{Z}}(Y)\right\rangle$ and $r=s$, then

$$
\begin{aligned}
& \Phi_{R}=\tilde{P}_{X} \Phi_{Y} \tilde{P}_{X}^{z} \\
& \Phi_{X}=P_{X} \Phi_{P} P_{X}^{*} .
\end{aligned}
$$


Proof. By (3.3) and (3.4)

$$
\Phi_{X}=\tilde{P}_{X} P=\tilde{P}_{X} \Phi_{Y}^{*} \tilde{P}_{X}^{*}
$$

which is (3.9), since $\Phi_{Y}$ is Hermitian. The relation (3.10) can be derived in the same manner.

Proof of Theorem 3.1. Let $y \in\left\langle T^{\mathrm{Z}}(Y)\right\rangle$ with $y \perp\left\langle T^{\mathrm{Z}}(X)\right\rangle$. Then for all $v \in \mathbf{Z}$ and $k=1, \ldots, s$,

$$
\begin{aligned}
0 & =\left\langle y, T^{v} x_{k}\right\rangle \\
& =\sum_{j=1}^{s} \sum_{n \in \mathbb{Z}}\left\langle y, T^{n} \tilde{y}_{j}\right\rangle\left\langle T^{n} y_{j}, T^{v} x_{k}\right\rangle \\
& =\sum_{j=1}^{s} \sum_{n \in \mathbb{Z}}\left\langle y, T^{n} \tilde{y}_{j}\right\rangle \overline{\left\langle T^{v-n} x_{k}, y_{j}\right\rangle}
\end{aligned}
$$

Let

$$
Y(\theta):=\left(\sum_{v \in Z}\left\langle y, T^{v} \tilde{y}_{j}\right\rangle e^{i v \theta}\right)_{j=1}^{s}
$$

Then $Y \in \tilde{L}_{s}^{2}(0,2 \pi)$. By $(3.11)$

$$
Y(\theta) P_{X}(\theta)^{*}=0
$$

Since $P_{X}(\theta)$ is invertible for almost all $\theta, Y(\theta)=0$ a.e. If follows from (3.12) that

$$
\left\langle y, T^{v} \tilde{y}_{j}\right\rangle=0, \quad v \in \mathbf{Z}, j=1, \ldots, s .
$$

Hence $y=0$.

Corollary 3.3. If $\left\langle T^{\mathrm{Z}}(X)\right\rangle \subset\left\langle T^{\mathrm{Z}}(Y)\right\rangle$ then $r \leqq s$.

Proof. Suppose $r>s$, and let $X^{\prime}=\left\{x_{1}, \ldots, x_{s}\right\}$. Then $T^{\mathrm{Z}}\left(X^{\prime}\right)$ is a Riesz basis of $\left\langle T^{\mathrm{Z}}\left(X^{\prime}\right)\right\rangle$ and $\left\langle T^{\mathrm{Z}}\left(X^{\prime}\right)\right\rangle \subset\left\langle T^{\mathrm{Z}}(Y)\right\rangle$. By Theorem 3.1, $\left\langle T^{\mathrm{Z}}\left(X^{\prime}\right)\right\rangle=\left\langle T^{\mathrm{Z}}(Y)\right\rangle$. But $T^{\mathrm{Z}}\left(X^{\prime}\right)$ is not complete in $\left\langle T^{\mathrm{Z}}(X)\right\rangle$. Hence there exists $x \neq 0$, such that $x \in\left\langle T^{\mathrm{Z}}(X)\right\rangle \subset\left\langle T^{\mathrm{Z}}(Y)\right\rangle$ and $x \perp\left\langle T^{\mathrm{z}}\left(X^{\prime}\right)\right\rangle=\left\langle T^{\mathrm{Z}}(Y)\right\rangle$, which is impossible.

Proof of Theorem 3.2. Suppose $\left\langle T^{\mathrm{Z}}(X)\right\rangle$ is a proper subspace of $\left\langle T^{\mathrm{Z}}(Y)\right\rangle$. By Theorem 3.1 and Corollary 3.3, $r<s$. Take any $\theta \in[0,2 \pi)$. The $r \times s$ matrix $P_{X}(\theta)=$ 
$\left(\sum_{n \in Z}\left\langle x_{k}, T^{n} y_{l}\right\rangle e^{i n \theta}\right)_{r \times s}$ has at most rank $r$. Hence there exists a unit vector $Y(\theta)=$ $\left(Y_{1}(\theta), \ldots, Y_{s}(\theta)\right) \in C^{s}$ (considered as a row vector) so that

$$
Y(\theta) P_{X}(\theta) * 0
$$

Since $\sum_{j=1}^{s}\left|Y_{j}(\theta)\right|^{2}=1$, the functions $Y_{j}$ thus defined are bounded functions in $\tilde{L}^{2}(0,2 \pi)$. Let

$$
Y_{j}(\theta)=\sum_{n \in \mathbf{Z}} a_{n}^{j} e^{i n \theta}
$$

where $\left(a_{n}^{j}\right)_{n \in Z} \in l^{2}(Z)$ for $j=1, \ldots, s$, and let

$$
x_{r+1}:=\sum_{j=1}^{s} \sum_{n \in Z} a_{n}^{j} T^{n} y_{j} .
$$

Then $x_{r+1} \in\left\langle T^{\mathbf{Z}}(Y)\right\rangle, a_{n}^{j}=\left\langle x_{r+1}, T^{n} \tilde{y}_{j}\right\rangle$ for $n \in \mathbf{Z}, j=1, \ldots, s$, and using the notation before Proposition 3.1,

$$
Y(\theta)=\widetilde{P}_{V}(\theta)
$$

where $V=\left\{x_{r+1}\right\}$. As in the proof of Theorem 3.1, (3.13) implies that

$$
\left\langle x_{r+1}, T^{v} x_{k}\right\rangle=0, \quad v \in \mathbf{Z}, k=1, \ldots, r .
$$

Hence $x_{r+1} \perp\left\langle T^{\mathrm{Z}}(X)\right\rangle$.

Since $T^{\mathrm{z}}(Y)$ is a Riesz basis of $\left\langle T^{\mathrm{z}}(Y)\right\rangle$, by Theorem 2.1, there exist positive constants $C_{1}$ and $C_{2}$, and a unitary matrix $U(\theta)$ such that

$$
\Phi_{Y}(\theta)=U(\theta) \operatorname{diag}\left(\lambda_{1}(\theta), \ldots, \lambda_{s}(\theta)\right) U(\theta)^{*}
$$

and

$$
C_{1} \leqq \lambda_{j}(\theta) \leqq C_{2}, \quad j=1, \ldots, s,
$$

for almost all $\theta$. By Proposition 3.1, for almost all $\theta$,

$$
\begin{aligned}
P_{V}(\theta) \tilde{P}_{V}(\theta)^{*} & =\tilde{P}_{V}(\theta) \Phi_{Y}(\theta) \tilde{P}_{V}(\theta)^{*} \\
& =\sum_{j=1}^{s} \lambda_{j}(\theta)|g(\theta)|^{2}
\end{aligned}
$$

where

$$
g(\theta)=\left(g_{1}(\theta), \ldots, g_{s}(\theta)\right)=\bar{P}_{V}(\theta) U(\theta)
$$


Since $\widetilde{P}_{V}(\theta)$ is a unit vector in $C^{s}$ and $U(\theta)$ in unitary, $\sum_{j=1}^{s}\left|g_{j}(\theta)\right|^{2}=1$. By (3.15) and (3.16),

$$
C_{1} \leqq P_{V}(\theta) \tilde{P}_{V}(\theta)^{*} \leqq C_{2} \quad \text { a.e.. }
$$

Hence the function $P_{V} \tilde{P}_{V}^{*}$ is a bounded function in $\tilde{L}^{2}(0,2 \pi)$, with $\left\langle x_{r+1}, T^{v} x_{r+1}\right\rangle$ as its $v$ th Fourier coefficient, for every $v \in \mathbf{Z}$. Therefore, $\left(\left\langle x_{r+1}, T^{v} x_{r+1}\right\rangle\right)_{v \in Z} \in l^{2}(Z)$ and if $\Phi_{v}(\theta):=\sum_{v \in Z}\left\langle x_{r+1}, T^{v} x_{r+1}\right\rangle e^{i v \theta}$, then

$$
\Phi_{V}(\theta)=P_{v}(\theta) \tilde{P}_{V}(\theta)^{*}
$$

By (3.17),

$$
C_{1} \leqq \Phi_{V}(\theta) \leqq C_{2} \quad \text { a.e. }
$$

Let $X^{\prime}=X \cup V$. By (3.14), we have

$$
\Phi_{X^{\prime}}(\theta)=\left(\begin{array}{cc}
\Phi_{X}(\theta) & 0 \\
0 & \boldsymbol{\Phi}_{V}(\theta)
\end{array}\right)
$$

Therefore the eigenvalues of $\Phi_{X}(\theta)$ comprise those of $\Phi_{X}(\theta)$ and $\Phi_{V}(\theta)$. Since $T^{\mathbf{z}}(X)$ is a Riesz basis of $\left\langle T^{\mathbf{z}}(X)\right\rangle$, by Theorem 2.1 and (3.18), $T^{\mathrm{Z}}\left(X^{\prime}\right)$ is a Riesz basis of $\left\langle T^{\mathbf{z}}\left(X^{\prime}\right)\right\rangle$.

Corollary 3.4. If $\left\langle T^{\mathrm{Z}}(X)\right\rangle$ is a proper subspace of $\left\langle T^{\mathrm{Z}}(Y)\right\rangle$ there exist $x_{r+1}, \ldots, x_{s}$ such that $T^{\mathrm{Z}}\left(X \cup\left\{x_{r+1}, \ldots, x_{s}\right\}\right)$ is a Riesz basis of $\left\langle T^{\mathrm{Z}}(Y)\right\rangle$. Furthermore

$$
\left\langle T^{\mathrm{z}}(Y)\right\rangle=\left\langle T^{\mathrm{Z}}(X)\right\rangle \oplus\left\langle T^{\mathrm{z}}\left\{x_{r+1}\right\}\right\rangle \oplus \cdots \oplus\left\langle T^{\mathrm{z}}\left\{x_{s}\right\}\right\rangle
$$

where $T^{\mathbf{z}}\left\{x_{j}\right\} \perp T^{\mathrm{Z}}(X)$ and $T^{\mathrm{z}}\left\{x_{j}\right\} \perp T^{\mathbf{z}}\left\{x_{l}\right\}$, for all $j, l=r+1, \ldots, s, j \neq l$.

Let $V$ be a finite subset of $\left\langle T^{\mathrm{z}}(Y)\right\rangle$. Using the notations introduced before Proposition 3.1, we give another characterization for $T^{\mathrm{Z}}(V)$ to be a Riesz basis of $\left\langle T^{\mathrm{Z}}(V)\right\rangle$.

Proposition 3.3. Let $V=\left\{v_{1}, \ldots, v_{m}\right\} \subset\left\langle T^{\mathrm{Z}}(Y)\right\rangle$, where $1 \leqq m \leqq s$. The following conditions are equivalent:

(1) $T^{\mathrm{Z}}(V)$ is a Riesz basis of $\left\langle T^{\mathrm{z}}(V)\right\rangle$.

(2) There exist positive constants $A$ and $B$ such that

$$
A I_{m} \leqq P_{V} P_{V}^{*} \leqq B I_{m} \quad \text { a.e.. }
$$

(3) There exist positive constants $\tilde{A}$ and $\widetilde{B}$ such that 


$$
\tilde{A} I_{m} \leqq \widetilde{P}_{V} \tilde{P}_{V}^{*} \leqq \tilde{B} I_{m} \text { a.e.. }
$$

Proof. Since $T^{\mathrm{Z}}(Y)$ is a Riesz basis of $\left\langle T^{\mathrm{Z}}(Y)\right\rangle$, by Theorem 2.1, there exist positive constants $C_{1}$ and $C_{2}$ such that

$$
C_{1} I_{s} \leqq \Phi_{Y} \leqq C_{2} I_{s} \quad \text { a.e.. }
$$

By (3.3),

$$
P_{V} P_{V}^{*}=\tilde{P}_{V} \Phi_{Y}^{2} \tilde{P}_{V}^{*}
$$

Hence by (3.19),

$$
C_{1}^{2} \tilde{P}_{V} \tilde{P}_{V}^{*} \leqq P_{V} P_{V}^{*} \leqq C_{2}^{2} \tilde{P}_{V} \tilde{P}_{V}^{*} \text { a.e., }
$$

which implies the equivalence of (2) and (3).

If $\left(\left\langle v_{k}, T^{n} v_{l}\right\rangle\right)_{n \in Z} \in l^{2}(Z), k, l=1, \ldots, m$, then by Proposition 3.1,

$$
\boldsymbol{\Phi}_{V}=\tilde{\boldsymbol{P}}_{\boldsymbol{V}} \boldsymbol{\Phi}_{Y} \tilde{P}_{\boldsymbol{V}}^{*}
$$

and so by (3.19),

$$
C_{1} \tilde{P}_{V} \tilde{P}_{V}^{*} \leqq \Phi_{V} \leqq C_{2} \tilde{P}_{V} \tilde{P}_{V}^{*}
$$

Therefore Theorem 2.1 and (3.21) give the implication $(1) \Rightarrow(3)$. Conversely, suppose that condition (2) holds (and so does (3)). These imply that all the entries in the matrices $P_{V}$ and $\widetilde{P}_{V}$ are bounded functions in $\tilde{L}^{2}(0,2 \pi)$. For $k, l=1, \ldots, m$, the $(k, l)$-entry of $\tilde{P}_{V} P_{v}^{*}$ is an $L^{2}$-function with $\left\langle v_{k}, T^{v} v_{l}\right\rangle$ as its $v$ th Fourier coefficient, for $v \in \mathbf{Z}$. Hence $\left(\left\langle v_{k}, T^{v} v_{l}\right\rangle\right)_{v \in Z} \in l^{2}(Z)$. Conditions (3.21), (3) and Theorem 2.1 then give (1).

\section{Wavelets in Hilbert space}

Let $T$ be a unitary operator on a complex Hilbert space $\mathscr{H}$, and suppose $X=\left\{x_{1}, \ldots, x_{r}\right\} \subset \mathscr{H}$ such that $T^{\mathrm{Z}}(X)$ is a Riesz basis of $V_{0}:=\left\langle T^{\mathrm{Z}}(X)\right\rangle$. Let $D$ be a unitary operator on $\mathscr{H}$ such that

$$
T D=D Y^{p}
$$

for some $p \in \mathbf{Z},|p|>1$, and

$$
V_{0} \subset V_{1}=: D V_{0}
$$

Then 


$$
\begin{aligned}
V_{1} & =\left\langle D\left(T^{\mathrm{Z}}(X)\right)\right\rangle \\
& =\left\langle\left\{T^{m} D T^{l} x_{j}: m \in \mathbf{Z}, \quad j=1, \ldots, r, l=0, \ldots,|p|-1\right\}\right\rangle,
\end{aligned}
$$

and $T^{\mathbf{z}}\left(\left\{D T^{l} x_{j}: j=1, \ldots, r, l=0, \ldots,|p|-1\right\}\right)$ is a Riesz basis of $V_{1}$. By Corollary 3.4 , there exists $\Gamma=\left\{z_{1}, \ldots, z_{r(|p|-1)}\right\} \in V_{1}$ such that $T^{\mathbf{Z}}\left\{z_{j}\right\} \perp V_{0}, T^{\mathbf{Z}}\left\{z_{j}\right\} \perp T^{\mathbf{Z}}\left\{z_{l}\right\}$ for $j \neq l$, $T^{\mathrm{z}}(X \cup \Gamma)$ is a Riesz basis of $V_{1}$ and

$$
V_{1}=V_{0} \oplus T^{\mathrm{z}}\left\{z_{1}\right\} \oplus \cdots \oplus T^{\mathrm{z}}\left\{z_{r(|p|-1)}\right\}
$$

Let $W_{0}$ be the orthogonal complement of $V_{0}$ in $V_{1}$. Then

$$
\begin{aligned}
W_{0} & =T^{\mathbf{z}}\left\{z_{1}\right\} \oplus \cdots \oplus T^{\mathbf{z}}\left\{z_{r(|p|-1)}\right\} \\
& =\left\langle T^{\mathbf{z}}(\Gamma)\right\rangle
\end{aligned}
$$

and $T^{\mathrm{z}}(\Gamma)$ is a Riesz basis of $W_{0}$.

We shall henceforward assume that $p=2$. In this case we have the following two-scale relations

$$
\begin{gathered}
x_{k}=\sum_{j=1}^{r} \sum_{n \in \mathbb{Z}}\left\langle x_{k}, D T^{n} \tilde{x}_{j}\right\rangle D T^{n} x_{j} \\
z_{k}=\sum_{j=1}^{r} \sum_{n \in Z}\left\langle z_{k}, D T^{n} \tilde{x}_{j}\right\rangle D T^{n} x_{j}, \quad k=1, \ldots, r,
\end{gathered}
$$

and decomposition relations

$$
D T^{l} x_{k}=\sum_{j=1}^{r} \sum_{n \in \mathbf{Z}}\left\{\left\langle D T^{l-2 n} x_{k}, \tilde{x}_{j}\right\rangle T^{n} x_{j}+\left\langle D T^{l-2 n} x_{k}, \tilde{z}_{j}\right\rangle T^{n} z_{j}\right\}, \quad l=0,1,
$$

where $\tilde{X}:=\left\{\tilde{x}_{1}, \ldots, \tilde{x}_{r}\right\}, \tilde{\Gamma}:=\left\{\tilde{z}_{1}, \ldots, \tilde{z}_{r}\right\}$, and $T^{\mathbf{z}}(\tilde{X})$ and $T^{\mathbf{Z}}(X)$ are biorthogonal Riesz bases of $V_{0}$, and $T^{\mathbf{z}}(\tilde{\Gamma})$ and $T^{\mathbf{z}}(\Gamma)$ are biorthogonal Riesz bases of $W_{0}$. Using the notations of Section 3, and letting

$$
Y:=\left\{D x_{j}, D T x_{j}: j=1, \ldots, r\right\},
$$

by (3.3) of Proposition 3.1, we can express (4.4) and (4.5) in the equivalent form

$$
P_{X \cup \Gamma}=\tilde{P}_{X \cup \Gamma} \Phi_{Y}
$$

Similarly by (3.7) or Corollary 3.1 , the decomposition relation (4.6) is equivalent to

$$
\Phi_{Y}=P_{X} \cup r P_{X \cup \Gamma}
$$


By Proposition 2.2 and Corollary 3.1, we have the dual relations

$$
\begin{aligned}
& \tilde{P}_{\bar{X} \cup \Gamma}=P_{X \cup \Gamma} \Phi_{\bar{P}} \\
& \Phi_{\mathcal{P}}=\tilde{P}_{X \cup \Gamma}^{*} \tilde{P}_{X \cup r} .
\end{aligned}
$$

The duality principle for the case of cardinal spline wavelets was first considered by Chui and Wang [3].

Let us now further assume that $r=1$, that is, there exists a vector $\phi$ in $\mathscr{H}$ such that $T^{\mathrm{Z}}(\{\phi\})$ is a Riesz basis of $V_{0}:=\left\langle T^{\mathrm{Z}}(\{\phi\})\right\rangle$,

$$
T D=D T^{2}
$$

and

$$
V_{0} \subset V_{1}:=D V_{0}
$$

Then there exists another vector $\bar{\phi}$ in $V_{0}$ such that $T^{\mathrm{Z}}(\{\tilde{\phi}\})$ is biorthogonal to $T^{\mathrm{Z}}(\{\phi\})$. Furthermore, letting

$$
\left\{\begin{array}{l}
\phi_{j}=D T^{j} \phi \\
\tilde{\phi}_{j}=D T^{j} \tilde{\phi}, \quad j=0,1,
\end{array}\right.
$$

then

$$
T^{\mathrm{Z}}\left(\left\{\phi_{0}, \phi_{1}\right\}\right)=\left\{D T^{n} \phi: n \in \mathbf{Z}\right\}
$$

and

$$
T^{\mathbf{Z}}\left(\left\{\tilde{\phi}_{0}, \tilde{\phi}_{1}\right\}\right)=\left\{D T^{n} \tilde{\phi}: n \in \mathbf{Z}\right\}
$$

are biorthogonal Riesz bases of $V_{1}$. The space $V_{1}$ can be expressed as

$$
\begin{aligned}
V_{1} & =\left\{g \in \mathscr{H}: g=\sum_{n \in \mathbf{Z}} \tilde{b}_{n} D T^{n} \phi, \quad \sum_{n \in \mathbf{Z}}\left|\tilde{b}_{n}\right|^{2}<\infty\right\} \\
& =\left\{g \in \mathscr{H}: g=\sum_{n \in \mathbf{Z}} b_{n} D T^{n} \tilde{\phi}, \quad \sum_{n \in \mathbf{Z}}\left|b_{n}\right|^{2}<\infty\right\} .
\end{aligned}
$$

Since $\phi$ and $\bar{\phi}$ are in $V_{1}$, they can be written as

$$
\begin{gathered}
\phi=\sum_{n \in \mathbf{Z}} \tilde{c}_{n} D T^{n} \phi, \quad \text { or } \\
\phi=\sum_{n \in \mathbf{Z}} c_{n} D T^{n} \tilde{\phi}, \quad \text { and }
\end{gathered}
$$




$$
\begin{gathered}
\tilde{\phi}=\sum_{n \in \mathbf{Z}} d_{n} D T^{n} \phi, \quad \text { or } \\
\bar{\phi}=\sum_{n \in \mathbf{Z}} d_{n} D T^{n} \tilde{\phi},
\end{gathered}
$$

where the filter coefficients $\left(c_{n}\right)_{n \in \mathbf{Z}},\left(\tilde{c}_{n}\right)_{n \in \mathbf{Z}},\left(d_{n}\right)_{n \in \mathbf{Z}},\left(d_{n}\right)_{n \in \mathbf{Z}}$ are in $l^{2}(\mathbf{Z})$.

Proposition 4.1. The filter coefficients satisfy

$$
\sum_{n \in \mathbf{Z}} \tilde{c}_{n-2 k} \bar{d}_{n}=\sum_{n \in \mathbf{Z}} c_{n-2 k} \bar{d}_{n}=\delta_{0, k}, \quad k \in \mathbf{Z}
$$

Proof. By (4.13)-(4.16),

$$
\left\langle T^{k} \phi, \Phi\right\rangle=\sum_{n \in Z} \tilde{c}_{n-2 k} \bar{d}_{n}=\sum_{n \in Z} c_{n-2 k} \widetilde{d}_{n}, \quad k \in \mathbf{Z} .
$$

The results follow since $\left\langle T^{k} \phi, \tilde{\phi}\right\rangle=\delta_{0, k}$.

A similar argument leads to:

Proposition 4.2. Let $f$ and $g$ be vectors in $V_{1}$, such that

$$
f=\sum_{n \in \mathbf{Z}} \tilde{a}_{n} D T^{n} \phi=\sum_{n \in \mathbf{Z}} a_{n} D T^{n} \tilde{\phi}
$$

and

$$
g=\sum_{n \in \mathbf{Z}} \tilde{b}_{n} D T^{n} \phi=\sum_{n \in \mathbf{Z}} b_{n} D T^{n} \tilde{\phi}
$$

Then $g$ is orthogonal to $\left\{T^{k} f: k \in \mathbf{Z}\right\}$

$$
\begin{array}{ll}
\Leftrightarrow \sum_{n \in \mathbf{Z}} \tilde{a}_{n-2 k} \overline{b_{n}}=0 & \text { for all } k \in \mathbf{Z} \\
\Leftrightarrow \sum_{n \in \mathbf{Z}} a_{n-2 k} \bar{b}_{n}=0 & \text { for all } k \in \mathbf{Z} .
\end{array}
$$

The orthogonal complement $W_{0}$ of $V_{0}$ in $V_{1}$ can be characterized as follows.

Proposition 4.3. The space $W_{0}$ is given by

$$
W_{0}=\left\{g \in \mathscr{H}: g=\sum_{n \in \mathbb{Z}} \tilde{b}_{n} D T^{n} \phi, \sum_{n \in \mathbf{Z}}\left|\tilde{b}_{n}\right|^{2}<\infty\right.
$$




$$
\left.\sum_{n \in \mathbf{Z}} c_{n-2 k} \widetilde{b_{n}}=0 \forall k \in \mathbf{Z} \text {, or } \sum_{n \in \mathbf{Z}} d_{n-2 k} \widetilde{b_{n}}=0 \forall k \in \mathbf{Z}\right\}
$$

and also by

$$
\begin{aligned}
W_{0}= & \left\{g \in \mathscr{H}: g=\sum_{n \in \mathbf{Z}} b_{n} D T^{n} \tilde{\phi}, \sum_{n \in \mathbf{Z}}\left|b_{n}\right|^{2}<\infty,\right. \\
& \left.\sum_{n \in \mathbf{Z}} \tilde{c}_{n-2 k} \bar{b}_{n}=0 \forall k \in \mathbf{Z}, \text { or } \sum_{n \in \mathbf{Z}} \widetilde{d}_{n-2 k} \bar{b}_{n}=0 \forall k \in \mathbf{Z}\right\} .
\end{aligned}
$$

Proof. Since $V_{0}=\left\langle T^{\mathrm{Z}}(\{\phi\})\right\rangle=\left\langle T^{\mathrm{Z}}(\{\delta\})\right\rangle$, the assertions follow from Proposition 4.2 and (4.12) to (4.16).

Let $g \in V_{1}$, and

$$
g=\sum_{n \in \mathbf{Z}} \tilde{b}_{n} D T^{n} \phi=\sum_{n \in \mathbb{Z}} b_{n} D T^{n} \tilde{\phi}
$$

where $\sum_{n \in Z}\left|\tilde{b}_{n}\right|^{2}<\infty$ and $\sum_{n \in Z}\left|b_{n}\right|^{2}<\infty$. Using the notations in Section 3, if $Y:=$ $\left\{\phi_{0}, \phi_{1}\right\}$ and $\tilde{Y}:=\left\{\tilde{\phi}_{0}, \tilde{\phi}_{1}\right\}$, then

$$
P_{\{g\}}(\theta)=\left(\sum_{n \in \mathbf{Z}} b_{2 n} e^{i n \theta}, \sum_{n \in \mathbf{Z}} b_{2 n+1} e^{i n \theta}\right)
$$

and

$$
\tilde{P}_{\{g\}}(\theta)=\left(\sum_{n \in Z} \tilde{b}_{2 n} e^{i n \theta}, \sum_{n \in Z} \tilde{b}_{2 n+1} e^{i n \theta}\right)
$$

Theorem 4.1. Let $g \in V_{1}$. The following conditions are equivalent:

(1) The set $T^{\mathrm{Z}}(\{g\})$ is a Riesz basis of $W_{0}$.

(2) The vector $g$ satisfies the condition in (4.20), and there exist positive constants $A$ and $B$ such that

$$
A \leqq P_{(g)}(\theta) P_{(g)}(\theta)^{*} \leqq B \quad \text { a.e. }
$$

(3) The vector $g$ satisfies the conditions in (4.19), and there exist positive constants $\tilde{A}$ and $\widetilde{B}$ such that

$$
\tilde{A} \leqq \tilde{P}_{\{g\}}(\theta) \tilde{P}_{\{g\}}(\theta)^{*} \leqq \widetilde{B} \quad \text { a.e.. }
$$


Proof. By Propositions 3.3 and 4.3 , we have $(1) \Rightarrow(2)$ and $(2) \Leftrightarrow(3)$. Conversely, suppose that (2) holds. This implies that $g \in W_{0}$, and $T^{\mathrm{Z}}(\{g\})$ is a Riesz basis of $\left\langle T^{\mathrm{Z}}(\{g\})\right\rangle$, which is a subset of $W_{0}$. Therefore we have

$$
\left\langle T^{\mathrm{Z}}(\{\phi, g\})\right\rangle=V_{0} \oplus\left\langle T^{\mathrm{z}}(\{g\})\right\rangle \subset V_{1}=\left\langle T^{\mathrm{z}}(Y)\right\rangle .
$$

By Theorem 3.1, the above inclusion is in fact an equality. Hence $\left\langle T^{\mathbf{z}}(\{g\})\right\rangle=W_{0}$ and so (1) holds.

The next theorem shows the construction of four vectors each of which satisfies the conditions in Theorem 4.1.

Theorem 4.2. With $\phi$ and $\Phi$ given by (4.13) to (4.16), let

$$
\begin{gathered}
\tilde{\eta}=\sum_{n \in \mathbf{Z}}(-1)^{-n+1} \overline{\tilde{c}_{-n+1}} D T^{n} \tilde{\phi}, \\
\psi=\sum_{n \in \mathbf{Z}}(-1)^{-n+1} \overline{c_{-n+1}} D T^{n} \phi, \\
\tilde{\psi}=\sum_{n \in \mathbf{Z}}(-1)^{-n+1} \overline{d_{-n+1}} D T^{n} \tilde{\phi}, \quad \text { and } \\
\eta=\sum_{n \in \mathbf{Z}}(-1)^{-n+1} \overline{d_{-n+1}} D T^{n} \phi .
\end{gathered}
$$

Then $T^{\mathbf{z}}(\{\tilde{\eta}\}), T^{\mathbf{Z}}(\{\psi\}), T^{\mathbf{z}}(\{\tilde{\psi}\})$ and $T^{\mathbf{z}}(\{\eta\})$ are all Riesz bases of $\boldsymbol{W}_{0}$. Furthermore, $T^{\mathrm{Z}}(\{\eta\})$ is biorthogonal to $T^{\mathrm{z}}(\{\tilde{\eta}\})$, and $\left.T^{\mathrm{z}}\{\psi\}\right)$ is biorthogonal to $T^{\mathrm{Z}}(\{\tilde{\psi}\})$.

Proof. We first prove that $T^{\mathbf{Z}}(\{\tilde{\eta}\})$ is a Riesz basis of $W_{0}$. For every $k \in \mathbf{Z}$,

$$
\begin{aligned}
& \sum_{n \in \mathbf{Z}}(-1)^{-n+1} \tilde{c}_{-n+1} \tilde{c}_{n-2 k}=\sum_{n \in \mathbf{Z}}\left(-\tilde{c}_{-2 n+1} \tilde{c}_{2 n-2 k}+\tilde{c}_{-2 n} \tilde{c}_{2 n+1-2 k}\right) \\
& =\sum_{n \in \mathbf{Z}}\left(-\tilde{c}_{-2 n+1} \tilde{c}_{2 n-2 k}+\tilde{c}_{-2 k+2 n} \tilde{c}_{-2 n+1}\right)=0 .
\end{aligned}
$$

By (4.20), $\tilde{\eta} \in W_{0}$. Since $T^{\mathrm{Z}}(\{\phi\})$ is a Riesz basis of $\left\langle T^{\mathrm{Z}}(\{\phi\})\right\rangle$, by Proposition 3.3, there exist positive constants $\tilde{A}$ and $\widetilde{B}$ such that

$$
\tilde{A} \leqq \widetilde{P}_{\{\phi\}}(\theta) \widetilde{P}_{\{\phi\}}(\theta)^{*} \leqq \tilde{B} \quad \text { a.e.. }
$$

By (4.13) and (4.22),

$$
\tilde{P}_{\{\phi\}}(\theta)=\left(\sum_{n \in \mathbf{Z}} \tilde{c}_{2 n} e^{i n \theta}, \sum_{n \in \mathbf{Z}} \tilde{c}_{2 n+1} e^{i n \theta}\right)
$$


By (4.23) and (4.21),

$$
P_{\{\tilde{\eta})}(\theta)=\left(-\sum_{n \in \mathrm{Z}} \overline{\tilde{c}_{-2 n+1}} e^{i n \theta}, \sum_{n \in \mathrm{Z}} \overline{\tilde{c}_{-2 n}} e^{i n \theta}\right)
$$

Hence

$$
P_{\{\tilde{r}\rangle}(\theta) P_{\{\tilde{r}\rangle}(\theta)^{*}=\left|\sum_{n \in \mathrm{Z}} \tilde{c}_{-2 n+1} e^{-i n \theta}\right|^{2}+\left|\sum_{n \in \mathrm{Z}} \tilde{c}_{-2 n} e^{-i n \theta}\right|^{2}=\tilde{P}_{\{\phi\rangle}(\theta) \tilde{P}_{\{\phi\rangle}(\theta)^{*}
$$

Then by (4.27), we have

$$
\tilde{A} \leqq P_{1 \tilde{\eta}}(\theta) P_{\{\tilde{\eta}}(\theta)^{*} \leqq \tilde{B} \quad \text { a.e.. }
$$

Hence by Theorem $4.1, T^{\mathrm{Z}}(\{\tilde{\eta}\})$ is a Riesz basis of $W_{0}$. The assertions involving $\psi, \tau$ and $\eta$ are proved similarly.

For every $k \in Z$, by (4.18) and (4.17),

$$
\begin{aligned}
\left\langle T^{k} \eta, \tilde{\eta}\right\rangle & =\sum_{n \in \mathbf{Z}}(-1)^{-n+2 k+1} \overline{d_{-n+2 k+1}}(-1)^{-n+1} \tilde{c}_{-n+1} \\
& =\sum_{n \in \mathbf{Z}} \tilde{c}_{-n+1} \overline{d_{-n+2 k+1}} \\
& =\sum_{n \in \mathbf{Z}} \tilde{c}_{n-2 k} \overline{d_{n}} \\
& =\delta_{0, k} .
\end{aligned}
$$

Hence $T^{\mathrm{z}}(\{\eta\})$ is biorthogonal to $T^{\mathrm{z}}(\langle\tilde{\eta}\})$. The proof for the case involving $\psi$ and $\Psi$ is similar.

We now consider the special case when $T^{\mathrm{Z}}(\{\phi\})$ is orthonormal.

Corollary 4.1. Let $T^{\mathrm{Z}}(\{\phi\})$ be an orthonormal basis of $V_{0}$, and let

$$
\phi=\sum_{n \in \mathbf{Z}} c_{n} D T^{n} \phi, \quad \sum_{n \in \mathbf{Z}}\left|c_{n}\right|^{2}<\infty
$$

Then

$$
\sum_{n \in \mathbf{Z}} c_{n-2 k} \overline{c_{n}}=\delta_{0, k} \quad k \in \mathbf{Z}
$$

and if 


$$
\psi=\sum_{n \in Z}(-1)^{-n+1} \overline{c_{-n+1}} D T^{n} \phi
$$

then $T^{\mathbb{Z}}(\{\psi\})$ is an orthonormal basis of $W_{0}$.

Proof. The assertions are easy consequences of Theorem 4.2.

Remark 2. The assertions in Corollary 4.1 can be proved directly without recourse to Fourier analysis.

Remark 3. For the case of orthonormal wavelet basis in $L^{2}(\mathbf{R})$, Corollary 4.1 is well known (see for example [5, 12, 13]).

We now give a general discussion on the decomposition and reconstruction formulae involving the scaling function $\phi$, wavelet functions $\psi$ and $\eta$, and their duals which are defined as in (4.13)-(4.16) and (4.23)- (4.26).

Suppose the $J$ th level "averages"

$$
s_{v}^{J}=\left\langle f, \phi_{J, v}\right\rangle, \quad v=0, \ldots, 2^{J}-1,
$$

of a vector $f$ are available. Here $\phi_{j, v}=D^{j} T^{v} \phi$.

One can then apply the following decomposition formulae

$$
\begin{gathered}
s_{v}^{j}=\sum_{l \in \mathbf{Z}} p_{l-2 v} s_{l}^{j+1}, \\
t_{v}^{j}=\sum_{l \in \mathbf{Z}} q_{l-2 v} v_{l}^{j+1}, \quad v=0, \ldots, 2^{j}-1
\end{gathered}
$$

for $j=J-1, \ldots, 0$ to obtain the "averages" $\left(s_{v}^{j}\right)$ and "details" $\left(t_{v}^{j}\right)$ for the next $J$ lower levels of resolution. The sequences $\left(p_{l}\right)_{l \in Z}$ and $\left(q_{l}\right)_{l \in Z}$ are the decomposition sequences. After some appropriate filtering processing of the pyramidal structure of the "details" and the "averages", one can recover $s_{v}^{J}$ through the use of the reconstruction formula

$$
s_{v}^{j+1}=\sum_{l \in \mathbf{Z}} a_{v-2 l} l_{l}^{j}+\sum_{l \in \mathbf{Z}} b_{v-2 l} t_{l}^{j}
$$

for $v=0, \ldots, 2^{j}-1, j=0, \ldots, J-1$. The sequences $\left(a_{l}\right)_{l \in \mathbf{Z}}$ and $\left(b_{l}\right)_{\epsilon \in \mathbf{Z}}$ are the reconstruction sequences.

The sequences $\left(p_{t}\right)_{\epsilon \in Z}$ and $\left(a_{t}\right)_{t \in Z}$ are obtained from the two-scale relations for $\phi$ and $\Phi$. Using (4.13) and (4.16), we have

$$
\begin{aligned}
& p_{l}=\overline{\tilde{c}_{l}}, \quad l \in \mathbf{Z}, \\
& a_{l}=d_{l}, \quad l \in \mathbf{Z} .
\end{aligned}
$$


The sequences $\left(q_{1}\right)_{l \in Z}$ and $\left(b_{l}\right)_{l \in Z}$ are obtained from the two-scale relations for the wavelet function and its dual. For $\psi$ and $\psi$, using (4.24) and (4.25), we obtain

$$
\begin{aligned}
& q_{l}=(-1)^{-l+1} c_{-l+1}, \quad l \in \mathbf{Z}, \\
& b_{l}=(-1)^{-l+1} \bar{d}_{-l+1}, \quad l \in \mathbf{Z} .
\end{aligned}
$$

For $\eta$ and $\tilde{\eta}$, using (4.23) and (4.26), we obtain

$$
\begin{aligned}
& q_{l}=(-1)^{-l+1} d_{-l+1}, \quad l \in \mathbf{Z}, \\
& b_{l}=(-1)^{-l+1} \bar{c}_{-l+1}, \quad l \in \mathbf{Z} .
\end{aligned}
$$

\section{Cardinal spline wavelets}

It is well known that the uniform B-spline $N_{k}$ of order $k$ defined by

$$
N_{1}=\chi_{[0,1)}
$$

and

$$
N_{k}=N_{k-1} * N_{1}, \quad k=2,3, \ldots,
$$

generates a multiresolution approximation $\left(V_{m}\right)_{m \in Z}$ of $L^{2}(\mathbf{R})([13,2])$. Indeed for a fixed $k, T^{\mathbf{Z}}\left\{N_{k}\right\}$ is a Riesz basis of $V_{0}:=\left\langle T^{\mathbf{Z}}\left\{N_{k}\right\}\right\rangle$ and $V_{m}:=D^{m} V_{0}$, where

$$
T f(x)=f(x-1)
$$

and

$$
D f(x)=\sqrt{2} f(2 x), \quad f \in L^{2}(\mathbf{R})
$$

By Lemma 2.1, $F: V_{0} \rightarrow V_{0}$ such that

$$
F(f)=\sum_{n \in \mathbf{Z}}\left\langle f, T^{n} N_{k}\right\rangle T^{n} N_{k}
$$

is a positive, invertible operator which commutes with $T$. A straightforward calculation gives

$$
F\left(\sum_{n \in \mathbf{Z}} a_{n} T^{n} N_{k}\right)=\sum_{n \in \mathbf{Z}}\left(\sum_{v \in \mathbf{Z}} a_{v} N_{2 k}(n+k-v)\right) T^{n} N_{k}
$$

The fundamental spline

$$
L_{2 k}(x)=\sum_{v \in \mathbf{Z}} \alpha_{v}^{(2 k)} N_{2 k}(x+k-v)
$$


satisfies

$$
L_{2 k}(n)=\delta_{0, n}
$$

(see $[15,2])$. It follows from $(5.6)$ that

$$
\tilde{N}_{k}:=F^{-1}\left(N_{k}\right)=\sum_{n \in Z} \alpha_{n}^{(2 k)} T^{n} N_{k}
$$

By the discussion following Lemma 2.1, $T^{\mathbf{z}}\left\{N_{k}\right\}$ and $T^{\mathbf{Z}}\left\{\tilde{N}_{k}\right\}$ are biorthogonal Riesz bases of $V_{0}$. This was obtained by Chui and Wang [2] by a direct computation.

We use the notations in Section 4 , with $\phi=N_{k}$. A Riesz basis for the orthogonal complement $W_{0}$ of $V_{0}$ in $V_{1}$ is not unique. By the well known relation

$$
N_{k}(x)=\sum_{n=0}^{k} 2^{-k+1}\left(\begin{array}{l}
k \\
n
\end{array}\right) N_{k}(2 x-n)
$$

and comparing coefficients with (4.13) we have

$$
\tilde{c}_{n}= \begin{cases}2^{-k+1 / 2}\left(\begin{array}{l}
k \\
n
\end{array}\right), & n=0, \ldots, k \\
0, & \text { otherwise }\end{cases}
$$

With the function $\tilde{\eta}$ defined by (4.23), a straight forward computation gives

$$
\tilde{\eta}(x)=2^{-k+1}(-1)^{k} L_{2 k}^{(k)}(2 x-1)
$$

which is a scalar multiple of the spline wavelet $\eta_{k}(x)=L_{2 k}^{(k)}(2 x-1)$ of Chui and Wang [2].

Using (5.8), we obtain

$$
c_{n}=\left\langle\phi, D T^{n} \phi\right\rangle= \begin{cases}2^{-k+1 / 2} \sum_{j=0}^{k}\left(\begin{array}{l}
k \\
j
\end{array}\right) N_{2 k}(k-n+j), & n=-k+1, \ldots, 2 k-1, \\
0, & \text { otherwise. }\end{cases}
$$

With the function $\psi$ defined by (4.24), (5.11) yields

$$
\psi(x)=-2^{-k+1} \sum_{v+0}^{2 k-2}(-1)^{v} N_{2 k}(v+1) N_{2 k}^{(k)}(2 x+v),
$$

which is related to the compactly supported spline wavelet

$$
\psi_{k}(x)=2^{-k+1} \sum_{v=0}^{2 k-2}(-1)^{v} N_{2 k}(v+1) N_{2 k}^{(k)}(2 x-v)
$$


of Chui and Wang [3] by $\psi(x)=-\psi_{k}(x+k-1)$.

By a similar computation, we obtain the sequences for the filter coefficients in (4.15) and (4.16), viz.

$$
d_{n}=2^{-k+1 / 2} \sum_{l \in \mathbf{Z}} \alpha_{l}^{(2 k)}\left(\begin{array}{c}
k \\
n-2 l
\end{array}\right), \quad n \in \mathbf{Z}
$$

and

$$
d_{n}=2^{-k+1 / 2} \sum_{l \in Z} \alpha_{l}^{(2 k)} \sum_{j=0}^{k}\left(\begin{array}{l}
k \\
j
\end{array}\right) N_{2 k}(k-2 l-j+n), \quad n \in \mathbf{Z}
$$

The functions $\psi$ and $\eta$ in (4.25) and (4.26) are then given by

$$
\begin{gathered}
\Psi(x)=2^{-k+1}(-1)^{k} \sum_{l \in Z} \alpha_{l}^{(2 k)} L_{2 k}^{(k)}(2 x-1+2 l), \\
\eta(x)=2^{-k+1} \sum_{v=1}^{2 k-1}(-1)^{v} N_{2 k}(v) \sum_{l \in Z} \alpha_{l}^{(2 k)} N_{2 k}^{(k)}(2 x-1+v-2 l) .
\end{gathered}
$$

Note that $\Psi(x)$ is related to the dual wavelet $\Psi_{k}(x)$ of Chui and Wang [3] by $\bar{\psi}(x)=-\bar{\psi}_{k}(x+k-1)$. By Theorem 4.2, $T^{\mathrm{z}}(\{\eta\})$ and $T^{\mathrm{z}}(\{\tilde{\eta}\})$ are biorthogonal Riesz bases of $W_{0}$, and likewise for $T^{\mathrm{Z}}(\{\psi\})$ and $T^{\mathrm{Z}}(\{\tilde{\psi}\})$.

Using the sequences in (5.9), (5.11), (5.13) and (5.14), the decomposition and reconstruction sequences for these two wavelets $\psi$ and $\eta$ can be obtained via (4.34)(4.39). In this case, the sequences $\left(c_{l}\right)_{l \in Z}$ and $\left(\tilde{c}_{l}\right)_{l \in Z}$ are finite while $\left(d_{l}\right)_{l \in Z}$ and $\left(\tilde{d}_{l}\right)_{l \in Z}$ are infinite sequences which depend on the B-spline coefficients $\left(\alpha_{l}^{(2 k)}\right)_{l \in Z}$ of the fundamental function $L_{2 k}$. The sequence $\left(\alpha_{l}^{(2 k)}\right)_{l \in Z}$ satisfies the infinite linear system of equations

$$
\sum_{l \in \mathbf{Z}} \alpha_{l}^{(2 k)} N_{2 k}(k-l+j)=\delta_{j, 0} . \quad j \in \mathbf{Z}
$$

and has the properties

$$
\alpha_{i}^{(2 k)}=\alpha_{-l}^{(2 k)}, \quad k \in \mathbf{Z}
$$

and

$$
\alpha_{i}^{(2 k)}=O\left(\left|r_{k}\right|^{-|| \mid}\right), \quad l \rightarrow \pm \infty
$$

where $r_{k}$ is the largest root less than -1 of the Euler-Frobenius polynomial of degree $2 k-2$ with coefficients $(2 k-1) ! N_{2 k}(j), j=1, \ldots, 2 k-1$ (see [15, p. 38]). The absolute value of $\alpha_{i}^{(2 k)}$ decreases exponentially with increasing $|l|$. In numerical computations, we truncate the sequence to $\left(\alpha_{i}^{(2 k)}\right)_{i=-M}^{M}$. This results in a finite section of the linear system (5.17) which is diagonal dominant and can be efficiently solved by exploiting the 
symmetric nature of the sequence. Consequently the other sequences which depend on $\left(\alpha_{l}^{(2 k)}\right)$ are also truncated accordingly.

Note here that we have two sets of decomposition and reconstruction formulae for use in numerical applications, one for the $\phi-\psi$ pair and the other for the $\phi-\eta$ pair. For each of the two sets of formulae, two out of the four decomposition and reconstruction sequences are finite. For the first set, this same feature is seen in [3]. However, for the second set, if one were to interchange the roles of $\eta$ and $\tilde{\eta}$ as would be the case in [2], only one out of the four sequences would be finite.

\section{Determination of $s_{v}$}

One of the advantages of using spline wavelets is the ease with which the "averages" $s_{v}^{J}$ can be computed from the data using the cardinal spline interpolant. Suppose that the values of a function $f \in L^{2}(\mathbf{R})$ are available at the points $2^{-J} v, v \in \mathbf{Z}$. Let

$$
f_{J}(x)=\sum_{v \in \mathbf{Z}} f\left(2^{-J} v\right) L_{k}\left(2^{J} x-v\right)
$$

be the cardinal spline interpolant of $f$, where $L_{k}(x)$ is the order $k$ fundamental spline and $k$ is assumed to be an even integer [15]. Obviously $f_{J}(x) \in V_{J}$ and we can express

$$
f_{\mathcal{J}}(x)=\sum_{n \in \mathbf{Z}} s_{n}^{\prime} \tilde{\phi}_{J, n}(x)
$$

where

$$
s_{n}^{J}=2^{-J / 2} \int_{-\infty}^{\infty} f_{\mathcal{J}}\left(2^{-J} x\right) \phi(x-n) d x
$$

A direct computation using (5.18) and the relations

$$
L_{k}(x)=\sum_{j \in \mathbf{Z}} \alpha_{j}^{(k)} N_{k}(x+k / 2-j),
$$

and

$$
N_{2 k}=N_{k} * N_{k}
$$

leads to

$$
S_{n}^{J}=2^{-J / 2} \sum_{v \in \mathbf{Z}} f\left(2^{-J} v\right) \sum_{j \in \mathbf{Z}} \alpha_{j}^{(k)} N_{2 k}(k / 2+v+j-n) .
$$

Remark 4. In [10], a periodized version of wavelet algorithms is applied to the transformation and compression of matrices. A linear system in which the matrix satisfies a discrete analogue of the Beylkin-Coifman-Rokhlin condition (see [1]) is 
transformed by the wavelet decomposition algorithm and compressed. An approximate solution of the original linear system is then recovered from the solution of the compressed system by the reconstruction algorithm. Numerical results show that a judicious choice of compactly supported non-orthonormal spline wavelets of Chui and Wang [3] and those constructed in this section performs better in matrix compression than the compactly supported wavelets of Daubechies [5]. A detailed discussion can be found in [10].

\section{REFERENCES}

1. G. Beylkin, R. Coifman and V. Rokhlin, Fast wavelet transforms and numerical algorithms I, Comm. Pure Appl. Math. 44 (1991), 141-183.

2. Charles K. Chui and Jian-zhong Wang, A cardinal spline approach to wavelets, Proc. Amer. Math. Soc. 113 (1991), 785-793.

3. Charles $\mathrm{K}$. Chui and Jan-zhong Wang, On compactly supported spline wavelets and a duality principle, Trans. Amer. Math. Soc. 330 (1992), 903-915.

4. Charles K. Chui and Jian-zhong Wang, a general framework of compactly supported splines and wavelets, J. Approx. Theory 71 (1992), 263-304.

5. I. Daubechies, Orthonormal bases of compactly supported wavelet, Comm. Pure Appl. Math. 41 (1988), 909-996.

6. I. Daubechies, The wavelet transform, time-frequency localization and signal analysis, IEEE Trans. Infor. Theory 36 (1990), 961-1005.

7. R. E. Edwards, Fourier series (Volume II, Holt, Rinehart and Winston, Inc., 1967).

8. T. N. T. Goodman, S. L. LeE and W. S. TANG, Wavelets in wandering subspaces, Trans. Amer. Math. Soc. 338 (1993), 639-654.

9. P. R. Halmos, A Hilbert space problem book, 2nd edition (Springer-Verlag, 1982).

10. S. L. Lee, H. H. TAN and W. S. TANG, Wavelet transformations and matrix compression (Proc. Internt. Conf. on Advances in Computational Mathematics, New Delhi, 1993).

11. P. G. Lemarié, Ondelettes a localisation exponentielles, J. Math. Pures Appl. 67 (1988), 227-236.

12. S. Mallat, Multiresolution approximations and wavelet orthonormal bases of $L^{2}(\mathbf{R})$, Trans. Amer. Math. Soc. 315 (1989), 69-87.

13. Y. MeYer, Ondelettes et functions splines (Seminaire Equations aux Derivees Partielles, Ecole Polytechnique, Paris, 1986).

14. J. B. Robertson, On wandering subspaces for unitary operators, Proc. Amer. Math. Soc. 16 (1965), 233-236.

15. I. J. Schoenberg, Cardinal spline interpolation (CBMS-NSF Series in Appl. Math. 12, SIAM Publ., Philadelphia, 1973).

16. R. M. Young, An introduction to nonharmonic Fourier series (Academic Press, 1980).

Department of Mathematics

National University of Singapore

10 Kent Ridge Crescent

SINGAPORE 0511 